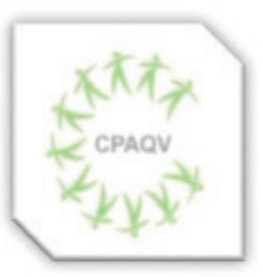

ISSN: 2178-7514

Vol. $12\left|\mathbf{N}^{\circ} .3\right|$ Ano 2020
ARTIGO ORIGINAL

\section{ATUAÇÃO FISIOTERAPÊUTICA NA REABILITAÇÃO DE DESARTICULAÇÃO METATARSOFALANGEANA TRAUMÁTICA: RELATO DE CASO}

Physiotherapeutic performance in the rehabilitation of traumatic metatarsophalangeal disarticulation: Case report

Joyce Lobato da Costa $^{1}$, Elissa Raissa Siqueira do Nascimento ${ }^{1}$, Cristian da Silva Costa ${ }^{1}$, Christian Pacheco de Almeida ${ }^{1}$, Andréa de Cassia Lima Guimarães ${ }^{2}$

\title{
RESUMO
}

Objetivo: Relatar a atuação fisioterapêutica na reabilitação de desarticulação metatarsofalangeana traumática. Métodos: Trata-se de um relato de experiência realizado durante o estágio curricular de Fisioterapia em Traumatologia e Ortopedia realizada na Unidade de Apoio ao Ensino em Fisioterapia e Terapia Ocupacional (UEAFTO), no ambulatório I do Centro Especializado em Reabilitação (CER-II), em fevereiro de 2020. O caso é de uma paciente em reabilitação após cirurgia de desarticulação metatarsofalangeana do hálux provocado por trauma automobilístico. Resultados: Durante a avaliação, foram identificadas alterações osteomioarticulares no pé esquerdo, alterações de marcha e equilíbrio. Além disso, observou-se quadro inflamatório local com edema característico. O tratamento fisioterapêutico durou 10 sessões. Conclusão: Durantes as 10 sessões houve redução dos sinais flogísticos de inflamação (dor, calor, edema, rubor, limitação na marcha). Da mesma forma, favoreceu o ganho de amplitude de movimento, possibilitou a descarga de peso e melhora de equilíbrio, contribuíram para o início do treino de marcha sem compensações, possibilitando a independência funcional.

Palavras-chave: Fisioterapia. Desarticulação. Reabilitação.

\section{ABSTRACT}

Objective: Report physiotherapeutic performance in the rehabilitation of traumatic metatarsophalangeal disarticulation. Methods: This is an experience report carried out during the curricular internship of Physiotherapy in Traumatology and Orthopedics carried out in the Teaching Support Unit in Physiotherapy and Occupational Therapy (UEAFTO), in ambulatory I of the Specialized Center for Rehabilitation (CER-II), in February 2020. The case is of a patient in rehabilitation after surgery for metatarsophalangeal dislocation of the hallux caused by car trauma. Results: During the 10 climbs, there was a reduction in the inflammatory signs of inflammation (pain, heat, edema, flushing, limited gait). Likewise, it favored gain in range of motion, enabled weight unloading, improved balance and contributed to the start of gait training without compensations, enabling functional independence. Conclusion: During the 10 sessions, the patient improved in relation to the reduction of inflammatory signs (pain, heat, edema, flushing, gait limitation). Likewise, it favored gain in range of motion, enable weight unloading and improve balance.

Keywords: Physiotherapy. Disarticulation. Rehabilitation

1 Graduandos em Fisioterapia da Universidade do Estado do Pará (UEPA)

2 Fisioterapeuta Mestra em Saúde, Sociedade e Endemias na Amazônia pela Universidade Federal do Pará (UFPA/UFAM/FIOCRUZ)

Autor de correspondência

Joyce Lobato da Costa

E-mail: joyce97cl@outlook.com 


\section{INTRODUÇÃO}

A desarticulação é definida como um procedimento cirúrgico de retirada parcialou total de algum membro para interromper o processo da afecção e evitar possíveis complicações ${ }^{1}$. Pode ter distintas etiologias, dentre essas estão: processos traumáticos, doenças vasculares, tumorais, infecciosas, congênitas e iatrogênicas, sendo a mais frequente as causas vasculares ${ }^{1,2}$.

É mais prevalente nos membros inferiores (MMII), correspondendo a cerca de 85\% das amputações. Ainda, seus fatores de risco incluem indivíduos com mais de 30 anos, do sexo masculino e pacientes com comorbidades relacionadas, a exemplo da diabetes mellitus ${ }^{2}$.

Nessa perspectiva, destaca-se a desarticulação metatarsofalangeana, ou seja, a retirada do contato do metatarso com as falanges3. Ademais, como o braço de alavanca é maior neste nível e devido ser uma região mais distal do MMII, ocorre menor impacto funcional com minimização das alterações de equilíbrio durante deambulação ${ }^{3}$.

Uma das causas para a ocorrência deste evento está relacionada a fatores traumáticos, principalmente, aqueles provenientes dos acidentes de trânsito e/ou ferimentos por arma de fogo. Sendo assim, quando estas situações ocorrem, o indivíduo passa a apresentar diversos impactos e complicações desde alterações psicossociais, tais como: alterações de imagem e esquema corporais, até distúrbios fisiológicos, como: fibrose no tecido cicatricial, perdas funcionais e alterações no ciclo da marcha, dentre outras ${ }^{4}$.

Vale ressaltar que, apesar dos impactos funcionais, em sua maioria, serem baixos, algumas complicações podem causar situações desagradáveis ao paciente e passam a ser indesejadas, dentre elas a dor e sensação fantasma, presença de contraturas e uma condição psicológica deprimida ${ }^{4}$.

Assim, destaca-se a atuação fisioterapêutica, a qual possui como principais objetivos minimizar as complicações instaladas, melhorando o edema do coto, diminuir e eliminar estados dolorosos, impedir contraturas articulares, prevenindo aderências e melhorar a independência e a qualidade de vida do paciente 5.

Diante disso, o objetivo do estudo foi relatar a atuação fisioterapêutica na reabilitação de desarticulação metatarsofalangeana traumática.

\section{MÉTODOS}

Trata-se de um estudo realizado durante o estágio curricular de Fisioterapia em Traumatologia e Ortopedia por alunos do $9^{\circ}$ semestre do curso de bacharelado em Fisioterapia, da Universidade do Estado do Pará (UEPA). A pesquisa foi desenvolvida na Unidade de Ensino Assistência em Fisioterapia e Terapia Ocupacional (UEAFTO), no ambulatório I do Centro Especializado em Reabilitação (CER-II), no mês de fevereiro de 2020.

Foi atendida, na ocasião, uma paciente 
do sexo feminino, 33 anos, praticante regular de atividade física (caminhada), encaminhada para a avaliação fisioterapêutica no mesmo mês em que se deu o estudo citado, após cirurgia de desarticulação metatarsofalangeana do hálux esquerdo, provocada por um acidente em via pública, em novembro de 2019. Durante a avaliação foi relatado pela participante como queixa principal a "dificuldade de andar" (alterações no ciclo da marcha) com predominância na perda de equilíbrio dinâmico.

\section{RESULTADOS}

$\mathrm{Na}$ história da doença atual, paciente relatou que durante dois meses não realizou descarga de peso, pois não se sentia segura em relação à deambulação. Ademais, por 20 dias utilizou dispositivo auxiliar de marcha (muletas) e, após esse período, iniciou a marcha com desconforto nos pé esquerdo, sensação e dor fantasma.

No exame físico, o pé esquerdo encontrava-se em evidente inversão, apresentando uma postura de proteção próxima ao local da cirurgia com limitação pelo edema residual no local. Foi realizado a perimetria na perna, e como referência para medição adotou-se a região abaixo da patela, dividindo de $6 \mathrm{em} 6 \mathrm{~cm}$, em terço proximal, terço médio e terço distal, apresentando como resultado para o lado direito: $31 \mathrm{~cm}, 28 \mathrm{~cm}$ e $22 \mathrm{~cm}$, respectivamente; e para o lado esquerdo: $32 \mathrm{~cm}, 29 \mathrm{~cm}, 23 \mathrm{~cm}$, respectivamente. Ao redor do tornozelo a medição foi realizada sobre os maléolos (medial e lateral), para o lado direito apresentou $21,0 \mathrm{~cm}$ e para o lado esquerdo 21,5 $\mathrm{cm}$. Além disso, foi realizado perimetria na região do médio pé, para o lado direito apresentou 20,5 $\mathrm{cm}$ e para o lado esquerdo $21,5 \mathrm{~cm}$.

Além do mais, foram observados os sinais flogísticos de inflamação (dor, calor, edema, rubor, limitação na marcha); calosidades na borda lateral e posterior do pé esquerdo; intolerância a superfícies ásperas na região em contato com o coto; dor à palpação na região do antepé, maléolo lateral e fáscie plantar com irradiação para o local da cirurgia, classificada em 3 (leve) de acordo com a Escala Visual Analógica (EVA), que é subjetiva para cada indivíduo.

Ainda na avaliação, verificaram-se limitações na amplitude de movimento (ADM) para os movimentos de flexão plantar, dorso flexão, inversão e eversão, levando a dificuldades na deambulação. Além disso, paciente apresentava alteração de equilíbrio estático e dinâmico, segundo o resultado positivo para o teste de Romberg,

Como também, apresentou diminuição da ADM no membro inferior esquerdo (MIE), com os seguintes achados: eversão com $3^{\circ}$; inversão com $7^{\circ}$; flexão plantar com $10^{\circ}$ e dorsiflexão com $15^{\circ}$, os quais foram verificados através do movimento ativo pelo goniômetro. Em comparação ao membro inferior direito (MIE), apresentou os seguintes achados: em eversão com $15^{\circ}$; inversão com $20^{\circ}$; flexão plantar com $28^{\circ}$ e dorsiflexão com $20^{\circ}$. 
Figura 1: Presença de edema na face medial do tornozelo em especial, ao entorno do maléolo medial.

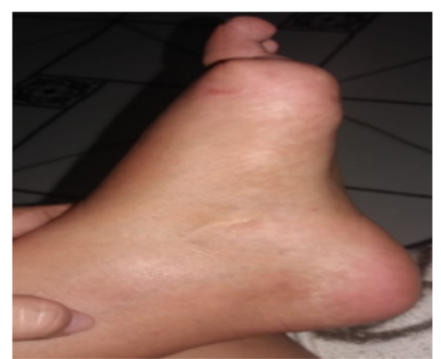

Fonte: Dos autores, sob autorização de paciente, 2020.

Figura 2: Presença de edema na face lateral do tornozelo e na região do retropé e médiopé.

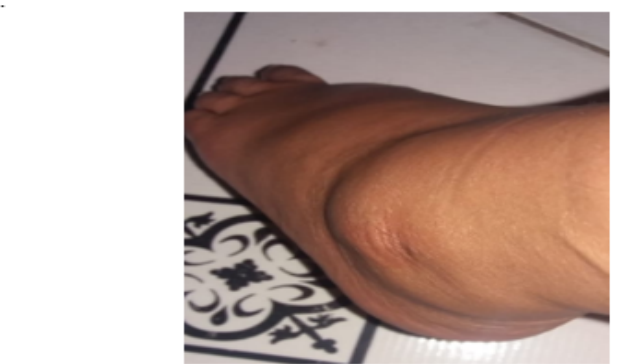

Fonte: Dos autores, sob autorização de paciente, 2020

No teste de força muscular, notou-se a redução de força para dorsiflexão e flexão plantar do pé esquerdo com grau 4 para ambos os movimentos, não sendo possível avaliar a força muscular em eversão e inversão devido edema e dor na região do tornozelo e antepé esquerdo.

$\mathrm{Na}$ aplicação do teste funcional Time Get Up And Go (TUG), a paciente realizou o percurso em 14 segundos, indicando risco moderado de quedas pela própria escala funcional. E na análise da marcha da mesma, apresentou marcha claudicante, pois durante a fase de apoio no membro esquerdo, paciente não realizava descarga total de peso, além de adotar uma postura de proteção inclinando o corpo lateralmente, provocando um padrão de marcha claudicante.

Baseando-se no conjunto de achados clínicos, a paciente apresentou problemas na marcha ligados diretamente ao equilíbrio, isto é, notória instabilidade. Seu diagnóstico cinético funcional foi descrito como limitação da amplitude de movimento (ADM) no tornozelo e pé esquerdo com alterações na marcha e no equilíbrio dinâmico.

O protocolo inicial de atendimento fisioterapêutico consistiu na aplicação de crioterapia por pacote na região do tornozelo esquerdo, com paciente em decúbito dorsal e elevação de membro durante 20 minutos a fim de diminuir o quadro álgico e o edema no antepé; exercício para facilitação de retorno venoso e drenagem linfática, com MMII elevados, apoiados sobre uma almofada em forma de triângulo, com paciente realizando movimentos de flexão plantar e dorsoflexão; alongamento ativo da musculatura 
do tríceps sural; drenagem linfática em toda perna até a fossa poplítea; dessensibilização por meio de massagem clássica da região próxima à cicatriz cirúrgica e finalizando com ultrassom $(1 \mathrm{MHz}$; 0,4 W/cm2 de potência; modo pulsado; por 4 minutos) na face anterior e lateral do tornozelo.

A partir da $4^{\circ}$ sessão, o tratamento evolui para uso de ultrassom (com os parâmetros anteriores mantidos); alongamentos ativos com uso de faixa elástica de cor rosa para dorsiflexão e flexão plantar; cinesioterapia ativa resistida com auxílio de bola para dorsiflexão; flexão plantar e eversão; descarga de peso em sedestação; e finalizando com crioterapia por 20 minutos.

A partir da $7^{\circ}$ sessão, acrescentou-se descarga de peso em bipedestação com exercício proprioceptivo e treino de marcha sobre colchões nas barras paralelas, compaciente treinandopassada. Exercício de descarga de peso e propriocepção em sedestação com paciente pressionando o pé sobre uma garrafa pet. Foram realizadas 100 sessões, com duração de 60 minutos, duas vezes por semana. Vale ressaltar que a paciente apresentava em sua guia de referência a liberação para a realização de $20^{\circ}$ sessões. Todavia, até a construção do presente relato, paciente encontrava-se na $10^{\circ}$ sessão, porém por se tratar de um estágio extracurricular, e da rotatividade dos acadêmicos nos setores, a paciente em questão teria o acompanhamento de outro terapeuta até completar o período previsto, após isso, será reavaliada para a verificação da necessidade do acréscimo ou não de mais sessões, até a alta completa da paciente.

Figura 3: Treino de descarga de peso em bipedestação

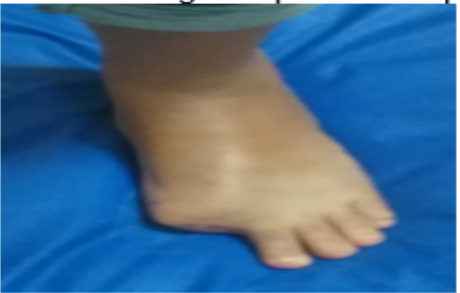

Fonte: Dos autores, sob autorização de paciente, 2020.

Figura 4: Exercício de descarga de peso e propriocepção em sedestação.

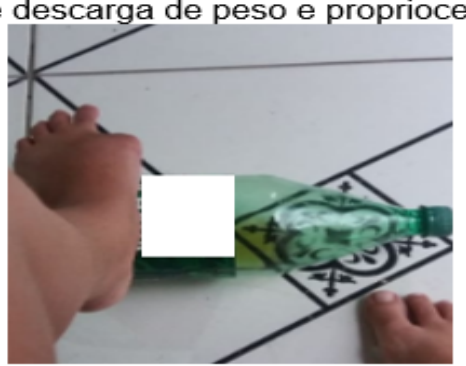

Fonte da Imagem: Dos autores, sob autorização de paciente, 2020. 
$\mathrm{Na} 10^{\circ}$ sessão, ocorreu a reavaliação, sendo evidenciado que após a intervenção fisioterapêutica ocorreu a redução dos sinais flogísticos da inflamação, na região do tornozelo, mas sem limitações ao movimento. Como também, a paciente referiu redução da sensação de dor fantasma, reduzindo de 3 para 0 de acordo com a escala EVA e melhora subjetiva relatada durante a locomoção.
Ademais, observou-se o aumento da ADM verificado com o goniômetro, para os movimentos ativos de flexão plantar $\left(21^{\circ}\right)$, dorsoflexão $\left(17^{\circ}\right)$, inversão $\left(20^{\circ}\right)$ e eversão $\left(11^{\circ}\right)$ para pé esquerdo. Assim como, melhora do contato do pé ao solo; melhora do equilíbrio; ganho de força muscular; diminuição da marcha claudicante; e no teste TUG paciente realizou o percurso em 9 segundos, indicando baixo risco de quedas pela classificação do próprio teste funcional.

Figura 4: Ilustração de pisada após descarga de peso em bipedestação, apresentando maior contato com o solo da região do antépé.

\section{DISCUSSÃO}

Segundo dados do Instituto Brasileiro de Geografia e Estatística (IBGE), no Brasil a quantidade de indivíduos com desarticulação aumentou significativamente nos últimos anos, com incidência média anual de 13,9 por 100 mil habitantes no país, com prevalência do sexo masculino - 58,2\% do total de amputações, contra $41,8 \%$ do sexo feminino ${ }^{6}$.

De acordo com a literatura, pessoas com amputações (definida como dissociação de um membro ou segmento) de MMII, apresentam piores prognósticos e redução considerável da

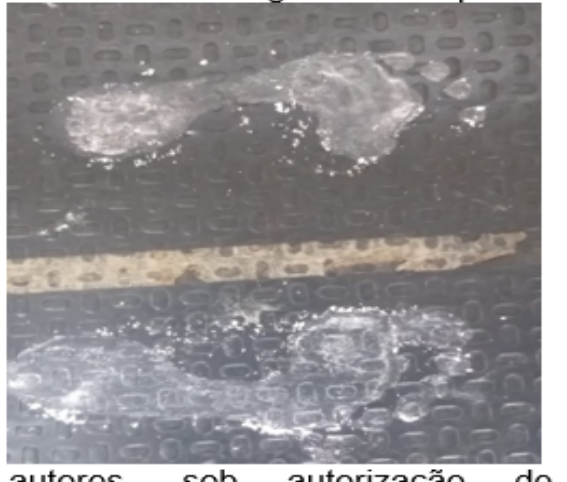

independência, pois não somente a funcionalidade é afetada, mas também a autonomia, autoestima, bem-estar e qualidade de vida ${ }^{7}$.

Nesse contexto, um dos maiores comprometimento físicos está relacionado à biomecânica da marcha, correspondendo as fases de balanço e apoio. Dessas, a fase de apoio é a mais prejudicada, pois para que ocorra de forma harmoniosa é necessário que haja sinergia dos centros superiores juntamente com informações periféricas para que o movimento seja realizado de forma satisfatória ${ }^{8}$.

Após o período pós-operatório, é notória a diferença entre o membro amputado e o membro 
preservado com redução dos sinais flogísticos, de modo que essa diferença influencia diretamente na postura e no tipo de marcha do individuo, vale ressaltar que o nível de amputação também apresenta influência sobre as estratégias posturais desenvolvidas. Assim como, a distribuição do peso corporal, além da posição dos eixos anteroposterior, laterolateral e a estabilização destes sujeitos. Nesse sentido, quanto mais alto o nível de amputação, mais alterações biomecânicas que o indivíduo pode apresentar ${ }^{8}$.

A paciente em questão, apresenta uma desarticulação (amputação que envolve a articulação) do tipo metatarsofalangeana do halúx esquerdo. E foi verificado alterações na fase de apoio, sugerindo uma adoção de postura de proteção, o que levou a um padrão de marcha claudicante, associado a episódios de desequilíbrio durante o balanceio, sendo esta sua queixa principal.

Frente a uma desarticulação, as fases da marcha são alteradas levando a disbasias. Isso é perceptível com a marcha claudicante que muitas vezes se instala logo após o processo cirúrgico. Além disso, a velocidade da marcha costuma também se alterar, fazendo com que ocorra um aumento no gasto energético, levando a comprometimento no desempenho funcional ${ }^{9}$.

Destaca-se ainda, a importância do cuidado do indivíduo após o processo de amputação com uma equipe multidisciplinar, em especial a intervenção fisioterapêutica, a qual demonstrou resultados consideráveis na melhora da funcionalidade e independência, corroborando com a literatura ${ }^{10}$.

Vale ressaltar, que o indivíduo que sofreu amputação no membro inferior, sofre com mudanças na circulação sanguínea local, alterações no esquema corporal associado ao equilíbrio e a marcha. Tal situação demanda adaptações quanto a realização de determinadas atividades, para isso é necessário a intervenção especializada de um profissional com enfoque de reabilitar este individuo ${ }^{11}$.

O início precoce da reabilitação pode minimizar os impactos da incapacidade gerados tanto pelo trauma, quanto pela processo cirúrgico e, assim, proporcionar um melhor prognóstico funcional ${ }^{11}$.

Ainda, a fisioterapia deve partir de uma avaliação global desde a inspeção até a aplicação de testes funcionais específicos para a elaboração do protocolo terapêutico. $\mathrm{Na}$ fase aguda, pode ser aplicado crioterapia, ultrassom e terapia manual para redução dos sinais flogísticos da inflamação ${ }^{12}$.

E nas fases subaguda e crônica, poderá ser utilizado a cinesioterapia, na qual os exercícios correspondem ao treinamento sistemático dos movimentos fisiológicos da região com o objetivo de aumentar a ADM e ganhar força muscular, o que a longo prazo promove melhora da marcha e consequentemente da funcionalidade. Esse prognóstico funcional favorável tem a finalidade de proporcionar maior independência e autonomia para o indivíduo ${ }^{13}$. 
$\mathrm{Na}$ paciente referida do estudo de caso, foi possível realizar uma avaliação completa e a partir do primeiro contato, traçar um protocolo terapêutico com base nos achados clínicos. E por meio das condutas aplicadas de forma individualizado ao caso, foram identificados ganhos significativos de ADM e força muscular; melhora da sensibilidade e do equilíbrio.

Resultados semelhantes ao encontrado na literatura que enfatiza a indicação da reabilitação fisioterapêutica o mais precoce possível para ganho de mobilidade e independência, promovendo ainda maior autoestima e qualidade de vida, visto que o procedimento de amputação é um processo que altera a vida da pessoa acometida14.

\section{CONCLUSÃO}

A realização do protocolo fisioterapêutico proposto durantes as 10 sessões apresentou resultados relevantes para a paciente, tais como a redução dos sinais flogísticos de inflamação (dor, calor, edema, rubor, limitação da marcha); favoreceu o ganho de amplitude de movimento; possibilitou a descarga de peso, fazendo com que o pé fica-se em maior contato com o solo melhorando assim o equilíbrio. Todos esses avanços contribuíram para o início do treino de marcha sem compensações, possibilitando a independência funcional.

Apesar do presente estudo não relatar o acompanhamento fisioterapêutico de forma precoce, no pós-cirúrgico, ainda foi possível perceber inúmeros benefícios durante as 10 sessões. Enfatiza-se assim, os ganhos funcionais promovidos durante a reabilitação pósdesarticulação metatarsofalangeana traumática com melhora da funcionalidade e qualidade de vida. E vale ressaltar a importância da continuidade ao tratamento, sempre com progressão para melhores resultados e preparação do seguimento corporal para receber prótese.

Este estudo, demonstra a importância do acompanhamento fisioterapêutico para um bom prognóstico funcional, tendo em vista a necessidade precoce de reabilitação com objetivo de prevenir incapacidades que podem se tornar irreversíveis. Sugerem-se que novos estudos possam ser realizados para verificar os benefícios funcionais ao indivíduo após a reabilitação fisioterapêutica de forma precoce, tanto no pré como no pós-operatório para promoção de funcionalidade e maior qualidade de vida.

\section{REFERÊNCIAS}

1. Leite VNV, Luza LP, Dias SMS, Caviquioni TZ, Zampirolo ERF, Silva RD. Avaliação postural de sujeitos com amputação de membro inferior. Scient. Medic. 2019; 29(1): 1-9.

2. Spichler D, Miranda FJ, Spichler ES, Franco LJ. Amputações maiores de membros inferiores por doença arterial periférica e diabetes melito no município do Rio de Janeiro. J. Vasc.Bras. 2020; 3(2): 111-122.

3. Luz JP, Ruaro JA, Ruaro MB, Kerppers II, Melo SA, Fréz AR. Fisioterapia em pacientes com amputação transtibial: revisão sistemática. ConScientiae Saúde. 2016; 15(1): 154-160.

4. Santos ACBC, Lee DLHM. Complicações pósoperatórias em amputados de membros inferiores. Revista InterScientia. 2016; 2(3): 1-9

5. Vieira RI, Luz SCT, Santos KPB, Junior EG, Campos 
PVC. Intervenções fisioterapêuticas utilizadas em pessoas amputadas de membros inferiores pré e pósprotetização: Uma revisão sistemática. Acta Fisiátrica. 2017; 24(2): 98-104.

6. Balardina AL, Andrighettia S, Schimit VM, Cechetti F, Bonetti LV, Saccani R. Análise Cinemática Linear e Angular da Marcha em Pacientes Amputados Transfemorais Protetizados. J. Healt. Scienc. 2018; 20(2): 125-130.

7. Branco RLL, Santos KPB, Luz SCT, Honório GJS, Medeiros PA. Percepções de pacientes amputados hospitalizados: uma exploração qualitativa do período pós-operatório. Revis Interdisc Estud Saúd. 2019; 8(1): 263-276.

8. Leite VNV, Luza LP, Dias SMS et al. Avaliação postural de sujeitos com amputação de membro inferior. Scientia Medica. 2019; 29(1): 1-9.

9. Passos PC, Leite SP, Lessa KMR, Bezerra MRS. A importância da análise da marcha e seu uso nas ciências aplicadas à educação física e motricidade humana. Cader. Gradu-Ciênc. Biológ Saúd- UNIT. 2016; 3(2): 119-124.

10. Fonseca RDCN, Ferreira APCS, Moutinho TP, Rainha TTA. A qualidade de vida e o edema do amputado de membro inferior submetido à tratamento fisioterapêutico. HCE. 2017; 1(1): 13-17.

11. Neves, Marta Francisca Almeida. Eficácia da implementação precoce de um programa de reabilitação nos utentes submetidos a amputação do membro inferior no sucesso da protetização. 2017. Tese de Doutorado.74 f. Instituto Politécnico de Viseu, 2017.

12. Monteiro HC, Silva VDFA, Ferreira MB, Barbosa D, Martins CA, Foresti BB. Perfil dos pacientes amputados de membros inferiores atendidos por um centro de referência: estudo clínico e epidemiológico. Revist FisiSenectus. 2018; 6(1): 38-47.

13. Branco RL, Santos KPB, Luz SC. Promovendo a saúde da pessoa amputada: uma ação educativa chamada conversa no leito. Brazilian Journal of Occupational Therapy/Cadern. Brasileir. Terap. Ocup. 2017; 25(3): 641- 648.

14. Vasconcelos, Thiago Brasileiro. Avaliação da qualidade de vida de pacientes amputados transtibiais unilaterais antes e após a protetização. Fisioterap Bras. 2017; 12(4): 291-297.

OBSERVAÇÃO: Os autores declaram não existir conflitos de interesse de qualquer

natureza. 